\title{
EULERIAN GAUSSIAN BEAM METHOD FOR HIGH FREQUENCY WAVE PROPAGATION IN HETEROGENEOUS MEDIA WITH DISCONTINUITIES IN ONE DIRECTION*
}

\author{
DONGMING $\mathrm{WEI}^{\dagger}$ AND XU YANG
}

\begin{abstract}
This paper generalizes the Eulerian Gaussian beam method developed in [S. Jin, H. Wu and X. Yang, Commun. Math. Sci., 6, 995-1020, 2008] to compute the high frequency wave propagation in heterogeneous media with discontinuities in one direction. The discontinuities in the wave speed correspond to the interfaces at which the incoming waves can be partially transmitted and reflected. We propose proper interface conditions to capture the reflection and transmission of Gaussian beams. These interface conditions arise naturally from an elegant combination of a Hamiltonian preserving scheme proposed in [S. Jin and X. Wen, Commun. Math. Sci., 3, 285-315, $2005]$ and the Eulerian Gaussian beam formulation. Numerical examples are also given to show the performance of this method.
\end{abstract}

Key words. High frequency wave, heterogeneous media, Eulerian Gaussian beam method, interface condition.

AMS subject classifications. 81Q20, 65M99.

\section{Introduction}

We are interested in developing efficient numerical methods for computing high frequency wave propagation in heterogeneous media. Specifically we consider the following linear scalar wave equation:

$$
\partial_{t}^{2} u-c^{2}(\boldsymbol{x}) \Delta_{\boldsymbol{x}} u=0, \quad \boldsymbol{x}=\left(x_{1}, \cdots, x_{n}\right) \in \mathbb{R}^{n},
$$

with the WKB initial conditions,

$$
\left\{\begin{array}{l}
u_{0}(\boldsymbol{x})=A_{0}(\boldsymbol{x}) e^{\frac{i}{\varepsilon} S_{0}(\boldsymbol{x})}, \\
\partial_{t} u_{0}(\boldsymbol{x})=\frac{1}{\varepsilon} B_{0}(\boldsymbol{x}) e^{\frac{i}{\varepsilon} S_{0}(\boldsymbol{x})},
\end{array}\right.
$$

where $u$ is the wave field, $n$ is the dimensionality, and $\mathrm{i}=\sqrt{-1}$ is the imaginary unit. The small parameter $\varepsilon \ll 1$ characterizes the high frequency. For simplicity, we assume the local wave speed $c(\boldsymbol{x})$ contains a discontinuity in $x_{1}$ direction,

$$
c(\boldsymbol{x})=\left\{\begin{array}{l}
c_{1}(\boldsymbol{x}) \text { if } x_{1}<0, \\
c_{2}(\boldsymbol{x}) \text { if } x_{1}>0,
\end{array}\right.
$$

where $c_{1}$ and $c_{2}$ are smooth functions. The interface is located at $x_{1}=0$.

Wave propagation described by (1.1)-(1.2) is an important and interesting problem in tomography and exploration seismology, where the rock structure is usually

${ }^{*}$ Received: September 14, 2011; accepted (in revised form): April 4, 2012. Communicated by Olof Runborg.

${ }^{\dagger}$ Division of Applied Mathematics, Brown University, Providence, RI 02912, USA (weidongmmm@hotmail.com).

${ }^{\ddagger}$ Department of Mathematics, Courant Institute of Mathematical Sciences, New York University, New York, NY 10012, USA (xuyang@cims.nyu.edu). 
heterogeneous and presents discontinuities. Direct numerical methods encounter two difficulties: the high oscillation of the solution and the reflection and transmission of waves at interfaces (see figure 1.1). A large number of grid points are required to resolve either issue, which makes the total computational cost quite expensive.

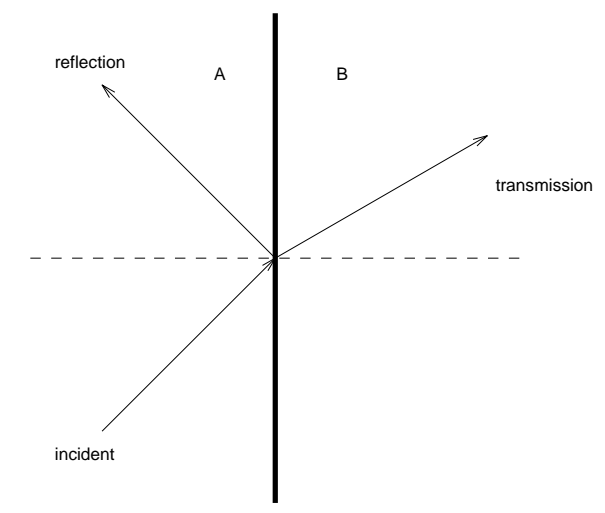

Fig. 1.1: Media $A$ and $B$ are different materials. The thick vertical line is the interface between them. The dashed line is the normal direction. An incident wave coming towards the interface in A will split into a reflection wave (in A) and a transmission wave (in B).

Alternative efficient methods are provided by asymptotic analysis. A famous example is geometric optics, which assumes the solution has the form

$$
u(t, x)=A(t, x) e^{\mathrm{i} S(t, x) / \varepsilon} .
$$

To leading order, the phase function $S$ satisfies the eikonal equation and the density $\rho$ satisfies the transport equation,

$$
\begin{aligned}
& \partial_{t} S+H\left(\boldsymbol{x}, \nabla_{\boldsymbol{x}} S\right)=0, \\
& \partial_{t} \rho+\nabla_{\boldsymbol{x}} \cdot\left(\rho \nabla_{\boldsymbol{\xi}} H\right)=0,
\end{aligned}
$$

where $\rho(t, \boldsymbol{x})=A^{2} / c^{2}$ and $H$ is the Hamiltonian function $H(\boldsymbol{x}, \boldsymbol{\xi})= \pm c(\boldsymbol{x})|\boldsymbol{\xi}|$. The " \pm " indicates the two wave branches.

The benefit of doing geometric optics is that one only needs to solve the macroscopic quantities $S$ and $A$, therefore the total numerical cost is independent of $\varepsilon$. However, there are two deficiencies:

1. The eikonal equation (1.5) is of Hamilton-Jacobi type, therefore the solution of (1.5) becomes singular after the formation of caustics. At caustics, the amplitude $A(t, x)$ blows up, which makes the approximate solution of geometric optics invalid $([2])$;

2. when $c(\boldsymbol{x})$ contains a discontinuity, (1.5)-(1.6) can not be applied directly due to the loss of regularity of the solution at the interface.

The first can be fixed by the Gaussian beam method, which was proposed by Popov $([1,20])$, analyzed by Ralston $([23])$, and later applied to seismic migration by Hill $([3,4])$. Recently, there have been a series of numerical studies on this method including higher order schemes, error estimates, Eulerian methods, and some new 
applications $[24,25,19,12,13,8,9,10,11,14,15,21,22]$. Moreover, frozen Gaussian approximation was introduced in $[16,17,18]$ to overcome the problem of the loss of accuracy when beams spread in the Gaussian beam method.

To overcome the second deficiency, one needs to apply Snell's Law of Refraction to capture the reflected and transmitted waves. The Hamiltonian preserving scheme proposed by Jin and Wen ([6]) naturally embeds Snell's Law of Refraction into the numerical flux of the Liouville equation. It efficiently computes wave propagation in heterogeneous media, but breaks down when caustics occur.

In this paper, we propose an Eulerian Gaussian beam method for computing wave propagation when the above two issues coexist. Our formulation is based on the Eulerian Gaussian beam method proposed in [8]. This method uses the first derivatives of level set functions to construct the Hessian matrix $M$, and it greatly reduces the number of equations needed in the traditional Eulerian Gaussian beam method $([12,13])$. We refer the readers to [5] for a more comprehensive review. When the local wave speed is discontinuous, proper conditions are required to correctly capture the reflection and transmission of the Gaussian beams. However, Snell's Law of Refraction only describes how the velocity and amplitude vary when the Gaussian beams hit the interface, while one still needs an extra interface condition for the Hessian function. The Hessian interface condition in the Lagrangian formulation has been studied by Weiss and Hagedorn on the wave equation with piecewise constant wave speed [27] and Yin and Zheng on one-dimensional Schrödinger equation [32]. Motivated by [6], we propose the interface conditions for the level set functions in the Eulerian Gaussian beam method, which preserves the Hamiltonian flow at the interface. The Hessian matrix is still constructed by the derivatives of level set functions as in [8]. We also derive the equivalent interface conditions in the Lagrangian formulation, which have been rigorously proved to provide the asymptotically accurate solution when the wave speed is piecewise constant ([27]).

The rest of the paper is organized as follows. In Section 2, we review the Eulerian Gaussian beam method for the scalar wave equation. In Section 3, we present the formulation and algorithm, and discuss the connection of the interface conditions to those used in the Lagrangian formulation [27]. In Section 4, we give some numerical examples to verify the accuracy of the method. We make conclusive remarks in Section 5.

\section{Eulerian Gaussian beam method}

The single Gaussian beam solution is given by,

$$
u(t, \boldsymbol{x})=A(t, \boldsymbol{y}) e^{\mathrm{i} \tilde{S}(t, \boldsymbol{x}, \boldsymbol{y}) / \varepsilon},
$$

with a complex phase function,

$$
\tilde{S}(t, \boldsymbol{x}, \boldsymbol{y})=S(t, \boldsymbol{y})+\boldsymbol{\xi}(t, \boldsymbol{y}) \cdot(\boldsymbol{x}-\boldsymbol{y})+\frac{1}{2}(\boldsymbol{x}-\boldsymbol{y}) \cdot M(t, \boldsymbol{y})(\boldsymbol{x}-\boldsymbol{y}),
$$

where the action $S \in \mathbb{R}$, the velocity $\boldsymbol{\xi} \in \mathbb{R}^{n}$, and the Hessian $M \in \mathbb{C}^{n \times n}$. Here $\boldsymbol{y}$ is called the beam center that follows the Hamiltonian flow given by $H_{ \pm}(\boldsymbol{x}, \boldsymbol{\xi})= \pm c(\boldsymbol{x})|\boldsymbol{\xi}|$,

$$
\left\{\begin{array}{c}
\frac{\mathrm{d} \boldsymbol{y}}{\mathrm{d} t}=\nabla_{\boldsymbol{\xi}} H_{ \pm}(\boldsymbol{y}, \boldsymbol{\xi}), \\
\frac{\mathrm{d} \boldsymbol{\xi}}{\mathrm{d} t}=-\nabla_{\boldsymbol{y}} H_{ \pm}(\boldsymbol{y}, \boldsymbol{\xi}) .
\end{array}\right.
$$


The imaginary part of $M$ is chosen to be positive definite so that the solution decays exponentially away from $\boldsymbol{x}=\boldsymbol{y}$. This makes the solution a Gaussian function. If the initial wave is not in the form of a single beam, one can approximate it by using a combination of Gaussian beams; see [25] for instance. The validity of this construction at caustics was analyzed by Ralston in [23].

The study of Eulerian Gaussian beam method was initiated by Leung, Qian, and Burridge in [12]. Later Jin, Wu, and Yang proposed a new formulation which greatly reduced the number of Liouville equations needed in the method. Here we briefly summarize the new formulation for the wave equation (1.1) with smooth local wave speed, and refer the readers to $[8,14]$ for more details. In the Eulerian formulation, $\boldsymbol{y}$ and $\boldsymbol{\xi}$ are discretized as mesh grids, and the structure of the Hamiltonian flow (2.2) goes into the Liouville operator,

$$
\mathcal{L}_{ \pm}:=\partial_{t}+\nabla_{\boldsymbol{\xi}} H_{ \pm} \cdot \nabla_{\boldsymbol{y}}-\nabla_{\boldsymbol{y}} H_{ \pm} \cdot \nabla_{\boldsymbol{\xi}}
$$

Here " \pm " corresponds to the two wave branches.

The phase function $S_{ \pm}(t, \boldsymbol{y}, \boldsymbol{\xi})$ and the amplitude function $A_{ \pm}(t, \boldsymbol{y}, \boldsymbol{\xi})$ satisfy

$$
\begin{aligned}
& \mathcal{L}_{ \pm} S_{ \pm}=0 \\
& \mathcal{L}_{ \pm} A_{ \pm}=\frac{A_{ \pm}}{2 H_{ \pm}}\left(\nabla_{\boldsymbol{\xi}} H_{ \pm} \cdot \nabla_{\boldsymbol{y}} H_{ \pm}+\nabla_{\boldsymbol{\xi}} H_{ \pm} \cdot\left(M_{ \pm} \nabla_{\boldsymbol{\xi}} H_{ \pm}\right)-c^{2}(\boldsymbol{y}) \operatorname{Tr}\left(M_{ \pm}\right)\right)
\end{aligned}
$$

with the initial conditions

$$
\begin{aligned}
& S_{ \pm}(0, \boldsymbol{y}, \boldsymbol{\xi})=S_{0}(\boldsymbol{y}) \\
& A_{ \pm}(0, \boldsymbol{y}, \boldsymbol{\xi})=\frac{1}{2}\left(A_{0}(\boldsymbol{y}) \pm \frac{\mathrm{i} B_{0}(\boldsymbol{y})}{c(\boldsymbol{y})\left|\nabla_{\boldsymbol{y}} S_{0}(\boldsymbol{y})\right|}\right)
\end{aligned}
$$

The level set functions

$$
\phi_{ \pm}(t, \boldsymbol{y}, \boldsymbol{\xi})=\left(\phi_{ \pm, 1}, \cdots, \phi_{ \pm, n}\right)
$$

are given by

$$
\mathcal{L}_{ \pm} \phi_{ \pm}=0
$$

with the initial condition

$$
\phi_{ \pm}=-\mathrm{i} \boldsymbol{y}+\left(\boldsymbol{\xi}-\nabla_{\boldsymbol{y}} S_{0}\right) .
$$

According to [8], the Hessian function $M_{ \pm}(t, \boldsymbol{y}, \boldsymbol{\xi})$ is constructed by

$$
M_{ \pm}=-\nabla_{\boldsymbol{y}} \phi_{ \pm}\left(\nabla_{\boldsymbol{\xi}} \phi_{ \pm}\right)^{-1}
$$

The solution to (1.1)-(1.2) is approximated by the following Eulerian Gaussian beam summation formula:

$$
\begin{aligned}
u_{G B}(t, \boldsymbol{x})= & \int_{\mathbb{R}^{2 n}}\left(\frac{1}{2 \pi \varepsilon}\right)^{\frac{n}{2}} r_{\theta}(|\boldsymbol{x}-\boldsymbol{y}|) \\
& \times\left(u_{+}(t, \boldsymbol{x}, \boldsymbol{y}, \boldsymbol{\xi}) \prod_{j=1}^{n} \delta\left(\operatorname{Re} \phi_{+, j}\right)+u_{-}(t, \boldsymbol{x}, \boldsymbol{x}, \boldsymbol{\xi}) \prod_{j=1}^{n} \delta\left(\operatorname{Re} \phi_{-, j}\right)\right) \mathrm{d} \boldsymbol{\xi} \mathrm{d} \boldsymbol{y},
\end{aligned}
$$


where $r_{\theta} \in C_{0}^{\infty}\left(\mathbb{R}^{n}\right), r_{\theta} \geq 0$ is a truncation function with $r_{\theta} \equiv 1$ in a ball of radius $\theta>0$ about the origin, $\delta$ is the Dirac delta function, and

$$
\begin{aligned}
& u_{ \pm}(t, \boldsymbol{x}, \boldsymbol{y}, \boldsymbol{\xi}) \\
= & A_{ \pm}(t, \boldsymbol{y}, \boldsymbol{\xi}) \times \exp \left(\frac{\mathrm{i}}{\varepsilon}\left(S_{ \pm}(t, \boldsymbol{y}, \boldsymbol{\xi})+\boldsymbol{\xi}(t, \boldsymbol{y}) \cdot(\boldsymbol{x}-\boldsymbol{y})+\frac{1}{2}(\boldsymbol{x}-\boldsymbol{y}) \cdot M_{ \pm}(t, \boldsymbol{y}, \boldsymbol{\xi})(\boldsymbol{x}-\boldsymbol{y})\right)\right) .
\end{aligned}
$$

\section{Formulation and algorithm}

In this section, we systematically introduce the formulation and algorithm of the Eulerian Gaussian beam method for the wave equation (1.1) with a discontinuous wave speed (1.3). Afterwards, we discuss the connection of the interface conditions to those used in the Lagrangian formulation.

3.1. Formulation. We first introduce several notations:

$$
\begin{aligned}
& \text { Region 1: }\left\{\boldsymbol{y} \mid y_{1}<0\right\}, \quad \boldsymbol{y}^{-}:=\left(0^{-}, y_{2}, \cdots, y_{n}\right), \quad C_{1}\left(\boldsymbol{y}^{-}\right):=\lim _{y_{1} \rightarrow 0^{-}} c_{1}(\boldsymbol{y}) ; \\
& \text { Region 2: }\left\{\boldsymbol{y} \mid y_{1}>0\right\}, \quad \boldsymbol{y}^{+}:=\left(0^{+}, y_{2}, \cdots, y_{n}\right), \quad C_{2}\left(\boldsymbol{y}^{+}\right):=\lim _{y_{1} \rightarrow 0^{+}} c_{2}(\boldsymbol{y}) ; \\
& \text { Transmission set from } j \text { to } k: \quad \Xi_{t r}^{j, k}:=\left\{\boldsymbol{\xi} \mid \frac{C_{j}^{2}}{C_{k}^{2}} \xi_{1}^{2}+\left(\frac{C_{j}^{2}}{C_{k}^{2}}-1\right) \sum_{l=2}^{n} \xi_{l}^{2}>0\right\} ; \\
& \text { Reflection set from } j \text { to } k: \quad \Xi_{r}^{j, k}:=\left\{\boldsymbol{\xi} \mid \frac{C_{j}^{2}}{C_{k}^{2}} \xi_{1}^{2}+\left(\frac{C_{j}^{2}}{C_{k}^{2}}-1\right) \sum_{l=2}^{n} \xi_{l}^{2}<0\right\} ; \\
& \text { Incident wave vector: } \quad \boldsymbol{\xi}^{-}:=\left(\xi_{1}^{-}, \xi_{2}, \ldots, \xi_{n}\right) ; \\
& \text { Transmitted wave vector: } \quad \boldsymbol{\xi}^{+}:=\left(\xi_{1}^{+}, \xi_{2}, \ldots, \xi_{n}\right) ; \\
& \text { Reflected wave vector: } \quad \boldsymbol{\xi}_{r}^{ \pm}:=\left(-\xi_{1}^{ \pm}, \xi_{2}, \ldots, \xi_{n}\right) .
\end{aligned}
$$

Snell's Law of Refraction implies

$$
C_{1}\left|\boldsymbol{\xi}^{-}\right|=C_{2}\left|\boldsymbol{\xi}^{+}\right|
$$

which is the conservation of the Hamiltonian $H_{ \pm}= \pm c(\boldsymbol{y})|\boldsymbol{\xi}|$ at the interface $y_{1}=0$.

Our method is motivated by the Hamiltonian preserving scheme [6]. The phase, amplitude and Hessian functions are solved by the Liouville equations (2.4), (2.5), and (2.6). We correct the flux at the interface by proper reflection and transmission conditions. This leads to an important reformulation of the level set functions (2.6): when the wave reaches the interface, the level sets given in (2.6) become discontinuous and hard to be computed due to the interaction of waves ([26]), and it turns out we need more than one set of level set functions to describe the reflected and transmitted beams.

In order to give a simple and clear presentation of the idea, we assume the initial wave comes from the left of the interface, and hit the interface only once. In this case, we need two sets of level set functions $\phi_{ \pm}^{r, t r}$, phase functions $S_{ \pm}^{r, t r}$, and amplitude functions $A_{ \pm}^{r, t r}$. The $t r$-system captures the incident wave and transmitted wave, and the $r$-system captures the incident wave and reflected wave. The general idea is to separate the propagation of reflected and transmitted waves by using respective level set functions, in order to avoid the interaction of different types of waves. The equation of $A_{ \pm}^{t r}$ describes the propagation of the wave amplitude of the incident and transmitted wave; the equation of $A_{ \pm}^{r}$ describes the propagation of the wave amplitude of the 
incident and reflected wave. The contribution of these functions to the final Gaussian beam solution will be described by the level set functions $\phi_{ \pm}^{r, t r}$. This is essentially the same idea as introduced in [26] for computing the semiclassical limit of the Schrödinger equation with discontinuous potentials. The details of the formulations for $S, A$, and $\phi$ are given as below.

The level set functions $\phi_{ \pm}^{r, t r}$ are solved by the following Liouville equations with the corresponding interface conditions at $y_{1}=0$ :

$$
\begin{aligned}
& \left\{\begin{array}{l}
\mathcal{L}_{ \pm} \boldsymbol{\phi}_{ \pm}^{r}(t, \boldsymbol{y}, \boldsymbol{\xi})=0 \\
\boldsymbol{\phi}_{ \pm}^{r}(0, \boldsymbol{y}, \boldsymbol{\xi})=-\mathrm{i} \boldsymbol{y}+\left(\boldsymbol{\xi}-\nabla_{\boldsymbol{y}} S_{0}\right) \\
\boldsymbol{\phi}_{ \pm}^{r}\left(t, \boldsymbol{y}^{-}, \boldsymbol{\xi}^{-}\right)=\boldsymbol{\phi}_{ \pm}^{r}\left(t, \boldsymbol{y}^{-}, \boldsymbol{\xi}_{r}^{-}\right)
\end{array}\right. \\
& \left\{\begin{array}{l}
\mathcal{L}_{ \pm} \boldsymbol{\phi}_{ \pm}^{t r}(t, \boldsymbol{y}, \boldsymbol{\xi})=0 \\
\boldsymbol{\phi}_{ \pm}^{t r}(0, \boldsymbol{y}, \boldsymbol{\xi})=-\mathrm{i} \boldsymbol{y}+\left(\boldsymbol{\xi}-\nabla_{\boldsymbol{y}} S_{0}\right) \\
\boldsymbol{\phi}_{ \pm}^{t r}\left(t, \boldsymbol{y}^{+}, \boldsymbol{\xi}^{+}\right)=\mathrm{I}_{\boldsymbol{\xi}^{-} \in \Xi_{t r}^{1,2}} \boldsymbol{\phi}_{ \pm}^{t r}\left(t, \boldsymbol{y}^{-}, \boldsymbol{\xi}^{-}\right)
\end{array}\right.
\end{aligned}
$$

where I is the characteristic function. The role of the characteristic function is to capture the waves that are admissible to be transmitted.

The phase functions $S_{ \pm}^{r, t r}$ are given by

$$
\begin{aligned}
& \left\{\begin{array}{l}
\mathcal{L}_{ \pm} S_{ \pm}^{r}(t, \boldsymbol{y}, \boldsymbol{\xi})=0 \\
S_{ \pm}^{r}(0, \boldsymbol{y}, \boldsymbol{\xi})=S_{0}(\boldsymbol{y}) \\
S_{ \pm}^{r}\left(t, \boldsymbol{y}^{-}, \boldsymbol{\xi}^{-}\right)=S_{ \pm}^{r}\left(t, \boldsymbol{y}^{-}, \boldsymbol{\xi}_{r}^{-}\right)
\end{array}\right. \\
& \left\{\begin{array}{l}
\mathcal{L}_{ \pm} S_{ \pm}^{t r}(t, \boldsymbol{y}, \boldsymbol{\xi})=0 \\
S_{ \pm}^{t r}(0, \boldsymbol{y}, \boldsymbol{\xi})=S_{0}(\boldsymbol{y}) \\
S_{ \pm}^{t r}\left(t, \boldsymbol{y}^{+}, \boldsymbol{\xi}^{+}\right)=\mathrm{I}_{\boldsymbol{\xi}^{-} \in \Xi_{t r}^{1,2}} S_{ \pm}^{t r}\left(t, \boldsymbol{y}^{-}, \boldsymbol{\xi}^{-}\right)
\end{array}\right.
\end{aligned}
$$

The amplitude functions $A_{ \pm}^{r, t r}$ are produced by

$$
\begin{aligned}
& \left\{\begin{array}{l}
\mathcal{L}_{ \pm} A_{ \pm}^{r}(t, \boldsymbol{y}, \boldsymbol{\xi})=\frac{A_{ \pm}^{r}}{2 H_{ \pm}}\left(\nabla_{\boldsymbol{\xi}} H_{ \pm} \cdot \nabla_{\boldsymbol{y}} H_{ \pm}+\nabla_{\boldsymbol{\xi}} H_{ \pm} \cdot\left(M_{ \pm} \nabla_{\boldsymbol{\xi}} H_{ \pm}\right)-c^{2}(\boldsymbol{y}) \operatorname{Tr}\left(M_{ \pm}\right)\right) \\
A_{ \pm}^{r}(0, \boldsymbol{y}, \boldsymbol{\xi})=\frac{1}{2}\left(A_{0}(\boldsymbol{y}) \pm \frac{\mathrm{i} B_{0}(\boldsymbol{y})}{c(\boldsymbol{y})\left|\nabla_{\boldsymbol{y}} S_{0}(\boldsymbol{y})\right|}\right) \\
A_{ \pm}^{r}\left(t, \boldsymbol{y}^{-}, \boldsymbol{\xi}^{-}\right)=R\left(\boldsymbol{\xi}^{-}\right) A_{ \pm}^{r}\left(t, \boldsymbol{y}^{-}, \boldsymbol{\xi}_{r}^{-}\right)
\end{array}\right. \\
& \left\{\begin{array}{l}
\mathcal{L}_{ \pm} A_{ \pm}^{t r}(t, \boldsymbol{y}, \boldsymbol{\xi})=\frac{A_{ \pm}^{t r}}{2 H_{ \pm}}\left(\nabla_{\boldsymbol{\xi}} H_{ \pm} \cdot \nabla_{\boldsymbol{y}} H_{ \pm}+\nabla_{\boldsymbol{\xi}} H_{ \pm} \cdot\left(M_{ \pm} \nabla_{\boldsymbol{\xi}} H_{ \pm}\right)-c^{2}(\boldsymbol{y}) \operatorname{Tr}\left(M_{ \pm}\right)\right) \\
A_{ \pm}^{t r}(0, \boldsymbol{y}, \boldsymbol{\xi})=\frac{1}{2}\left(A_{0}(\boldsymbol{y}) \pm \frac{\mathrm{i} B_{0}(\boldsymbol{y})}{c(\boldsymbol{y})\left|\nabla_{\boldsymbol{y}} S_{0}(\boldsymbol{y})\right|}\right) \\
A_{ \pm}^{t r}\left(t, \boldsymbol{y}^{+}, \boldsymbol{\xi}^{+}\right)=T\left(\boldsymbol{\xi}^{-}\right) A_{ \pm}^{t r}\left(t, \boldsymbol{y}^{-}, \boldsymbol{\xi}^{-}\right) .
\end{array}\right.
\end{aligned}
$$

The reflection coefficient $R\left(\boldsymbol{\xi}^{-}\right)$and transmission coefficient $T\left(\boldsymbol{\xi}^{-}\right)$are given by Snell's Law of Refraction,

$$
R\left(\boldsymbol{\xi}^{-}\right)=\left\{\begin{array}{ll}
-1 & \text { if } \boldsymbol{\xi}^{-} \in \Xi_{r}^{1,2}, \\
\frac{\xi_{1}^{-}-\xi_{1}^{+}}{\xi_{1}^{-}+\xi_{1}^{+}}, & \text {otherwise, }
\end{array} \quad T\left(\boldsymbol{\xi}^{-}\right)= \begin{cases}0 & \text { if } \boldsymbol{\xi}^{-} \in \Xi_{r}^{1,2} \\
\frac{2 \xi_{1}^{-}}{\xi_{1}^{-}+\xi_{1}^{+}}, & \text {otherwise }\end{cases}\right.
$$


Note that $1+R=T$. The first case in (3.5) corresponds to the total reflection, and the second corresponds to the partial reflection and transmission.

We define $u_{G B}^{r, t r}$ by replacing $S_{ \pm}, A_{ \pm}$, and $\phi_{ \pm}$with $S_{ \pm}^{r, t r}, A_{ \pm}^{r, t r}$, and $\phi_{ \pm}^{r, t r}$ in (2.8). Then the Gaussian beam solution for the discontinuous wave speed (1.3) is given by

$$
u_{G B}(t, \boldsymbol{x})=u_{G B}^{r}(t, \boldsymbol{x})+\mathrm{I}_{\left\{x_{1}>0\right\}} u_{G B}^{t r}(t, \boldsymbol{x}),
$$

where $u_{G B}^{r}(t, \boldsymbol{x})$ captures the wave that has not hit the interface and the reflected wave, and $\mathrm{I}_{\left\{x_{1}>0\right\}} u_{G B}^{t r}(t, \boldsymbol{x})$ captures the transmitted wave.

3.2. Algorithm. We describe the numerical algorithm as follows.

Step 1. Solve (3.2)-(3.3) using the Hamiltonian preserving scheme proposed in [6].

Step 2. Compute the Hessian function using the formulation (2.7).

Step 3. Solve the amplitude equation (3.4).

Step 4. Evaluate (3.6) by numerically discretizing the delta functions using the techniques given in $[28,29,30]$.

REMARK 3.1. If the initial wave comes from both sides of the interface, we can simply split it into left-coming and right-coming parts and apply the algorithm twice. For the case of multiple interfaces where a wave packet hits interfaces multiple times, we will need the method proposed in [26]. Briefly speaking, if the interfaces divide $\mathbb{R}^{n}$ into $K$ disjoint parts $\mathbb{R}^{n}=\bigcup_{j=1}^{K} \Omega_{j}$, one can decompose the original problem into $K+1$ systems, and the Gaussian beam solution takes the following form:

$$
u_{G B}(t, \boldsymbol{x})=u_{G B}^{r}(t, \boldsymbol{x})+\sum_{j=1}^{K} \mathrm{I}_{\left\{\mathbb{R}^{n} / \Omega_{j}\right\}} u_{G B}^{t r, j}(t, \boldsymbol{x}) .
$$

It is also necessary to reinitialize once between two successive hitting times of the same wave packet. Technically this is quite involved, and we will leave it for further study.

REMARK 3.2. The numerical method in Step 1 can also be replaced by a hybrid phase flow method introduced in [7,31]. In Step 2, the Hessian function can be easily computed by the Hamiltonian preserving scheme, since $\nabla_{y} \phi$ and $\nabla_{\xi} \phi$ are the numerical fluxes after discretizing the Liouville equations of $\phi$, as pointed out earlier in $[8]$.

3.3. Connection to the Lagrangian formulation. In this subsection, we explicitly derive the interface conditions for the Hessian functions from the Hamiltonian preserving conditions on $\phi$. This generalizes the results by Weiss and Hagedorn in [27].

Without loss of generality, we consider the "+" branch where $H(\boldsymbol{y}, \boldsymbol{\xi})=c(\boldsymbol{y})|\boldsymbol{\xi}|$. Then the interface conditions in (3.2) become,

$$
\begin{array}{lr}
\text { for reflection } & \boldsymbol{\phi}^{r}\left(t, \boldsymbol{y}^{-}, \boldsymbol{\xi}^{-}\right)=\boldsymbol{\phi}^{r}\left(t, \boldsymbol{y}^{-}, \boldsymbol{\xi}_{r}^{-}\right), \\
\text {for transmission } & \boldsymbol{\phi}^{t r}\left(t, \boldsymbol{y}^{-}, \boldsymbol{\xi}^{-}\right)=\boldsymbol{\phi}^{t r}\left(t, \boldsymbol{y}^{+}, \boldsymbol{\xi}^{+}\right) .
\end{array}
$$

We denote $M_{1}, M_{2}$, and $M_{3}$ as the Hessian functions for the incident, reflected and transmitted beams respectively. 
3.3.1. Hessian interface condition for the reflected beam. The condition (3.8) yields

$$
\nabla_{\boldsymbol{y}} \boldsymbol{\phi}^{r}\left(t, \boldsymbol{y}^{-}, \boldsymbol{\xi}^{-}\right)=\nabla_{\boldsymbol{y}} \boldsymbol{\phi}^{r}\left(t, \boldsymbol{y}^{-}, \boldsymbol{\xi}_{r}^{-}\right), \quad \nabla_{\boldsymbol{\xi}} \boldsymbol{\phi}^{r}\left(t, \boldsymbol{y}^{-}, \boldsymbol{\xi}^{-}\right)=F \nabla_{\boldsymbol{\xi}} \boldsymbol{\phi}^{r}\left(t, \boldsymbol{y}^{-}, \boldsymbol{\xi}_{r}^{-}\right),
$$

where $F$ is an $n \times n$ diagonal matrix with $F_{11}=-1$ and $F_{j j}=1, j \geq 2$. By (2.7), we obtain

$$
M_{2}=M_{1} F
$$

3.3.2. Hessian interface condition for the transmitted beam. The equation (3.1) yields

$$
\xi_{1}^{-}=\sqrt{\frac{C_{2}^{2}}{C_{1}^{2}}\left(\xi_{1}^{+}\right)^{2}+\left(\frac{C_{2}^{2}}{C_{1}^{2}}-1\right) \sum_{j=2}^{n} \xi_{j}^{2}}
$$

Then

$$
\begin{aligned}
& \partial_{\xi_{1}} \boldsymbol{\phi}^{t r}\left(t, \boldsymbol{y}^{+}, \boldsymbol{\xi}^{+}\right)= \lim _{\Delta \xi_{1} \rightarrow 0}\left(\phi^{t r}\left(t, \boldsymbol{y}^{+}, \xi_{1}^{+}+\Delta \xi_{1}, \xi_{2}, \cdots, \xi_{n}\right)\right. \\
&\left.-\boldsymbol{\phi}^{t r}\left(t, \boldsymbol{y}^{+}, \xi_{1}^{+}, \xi_{2}, \cdots, \xi_{n}\right)\right) \frac{1}{\Delta \xi_{1}} \\
&= \lim _{\Delta \xi_{1} \rightarrow 0}\left(\boldsymbol{\phi}^{t r}\left(t, \boldsymbol{y}^{-}, \sqrt{\frac{C_{2}^{2}}{C_{1}^{2}}\left(\xi_{1}^{+}+\Delta \xi_{1}\right)^{2}+\left(\frac{C_{2}^{2}}{C_{1}^{2}}-1\right) \sum_{j=2}^{n} \xi_{j}^{2}}, \xi_{2}, \cdots, \xi_{n}\right)\right. \\
&\left.-\boldsymbol{\phi}^{t r}\left(t, \boldsymbol{y}^{-}, \xi_{1}^{-}, \xi_{2}, \cdots, \xi_{n}\right)\right) \frac{1}{\Delta \xi_{1}} \\
&=\frac{C_{2}^{2}}{C_{1}^{2}} \frac{\xi_{1}^{+}}{\xi_{1}^{-}} \partial_{\xi_{1}} \boldsymbol{\phi}^{t r}\left(t, \boldsymbol{y}^{-}, \boldsymbol{\xi}^{-}\right) .
\end{aligned}
$$

Similarly, for $l \geq 2$,

$$
\partial_{\xi_{l}} \boldsymbol{\phi}^{t r}\left(t, \boldsymbol{y}^{+}, \boldsymbol{\xi}^{+}\right)=\left(\frac{C_{2}^{2}}{C_{1}^{2}}-1\right) \frac{\xi_{l}}{\xi_{1}^{-}} \partial_{\xi_{1}} \phi^{t r}\left(t, \boldsymbol{y}^{-}, \boldsymbol{\xi}^{-}\right)+\partial_{\xi_{l}} \boldsymbol{\phi}^{t r}\left(t, \boldsymbol{y}^{-}, \boldsymbol{\xi}^{-}\right) .
$$

Therefore

$$
\nabla_{\boldsymbol{\xi}} \boldsymbol{\phi}^{t r}\left(t, \boldsymbol{y}^{+}, \boldsymbol{\xi}^{+}\right)=G\left(\boldsymbol{\xi}^{-}\right) \nabla_{\boldsymbol{\xi}} \boldsymbol{\phi}^{t r}\left(t, \boldsymbol{y}^{-}, \boldsymbol{\xi}^{-}\right)
$$

where $G\left(\boldsymbol{\xi}^{-}\right)$is an $n \times n$ matrix given by: $G_{11}=\frac{C_{2}^{2}}{C_{1}^{2}} \frac{\xi_{1}^{+}}{\xi_{1}^{-}} ; G_{j 1}=\left(\frac{C_{2}^{2}}{C_{1}^{2}}-1\right) \frac{\xi_{j}}{\xi_{1}^{-}}, G_{j j}=1$, $j \geq 2$; all the other entries are zero.

Next, we derive the conditions of $\nabla_{\boldsymbol{x}} \phi^{t r}$ at the interface. The equation (3.9) implies

$$
\partial_{t} \boldsymbol{\phi}^{t r}\left(t, \boldsymbol{y}^{-}, \boldsymbol{\xi}^{-}\right)=\partial_{t} \boldsymbol{\phi}^{t r}\left(t, \boldsymbol{y}^{+}, \boldsymbol{\xi}^{+}\right) .
$$

Then the Liouville Equation (3.2) produces, at the interface,

$$
\left[\nabla_{\boldsymbol{\xi}} H \cdot \nabla_{\boldsymbol{y}} \boldsymbol{\phi}^{t r}\right]_{y_{1}=0}=\left[\nabla_{\boldsymbol{y}} H \cdot \nabla_{\boldsymbol{\xi}} \boldsymbol{\phi}^{t r}\right]_{y_{1}=0}
$$

where $[\cdot]$ means the jump function. 
Notice that for $j \geq 2$,

$$
\partial_{y_{j}} \phi^{t r}\left(t, \boldsymbol{y}^{-}, \boldsymbol{\xi}^{-}\right)=\partial_{y_{j}} \boldsymbol{\phi}^{t r}\left(t, \boldsymbol{y}^{+}, \boldsymbol{\xi}^{+}\right)
$$

Then the condition (3.12) implies

$$
\nabla_{\boldsymbol{y}} \boldsymbol{\phi}^{t r}\left(t, \boldsymbol{y}^{+}, \boldsymbol{\xi}^{+}\right)=W\left(\boldsymbol{\xi}^{-}\right) \nabla_{\boldsymbol{y}} \boldsymbol{\phi}^{t r}\left(t, \boldsymbol{y}^{-}, \boldsymbol{\xi}^{-}\right)+\frac{\left|\boldsymbol{\xi}^{+}\right|}{C_{2} \xi_{1}^{+}} \overrightarrow{e_{1}}\left[|\boldsymbol{\xi}| \nabla_{\boldsymbol{y}} c(\boldsymbol{y}) \cdot \nabla_{\boldsymbol{\xi}} \boldsymbol{\phi}^{t r}\right]_{y_{1}=0},
$$

where $\overrightarrow{e_{1}}=(1,0, \cdots, 0)^{\mathrm{T}}$, and $W\left(\boldsymbol{\xi}^{-}\right)$is an $n \times n$ matrix given by: $W_{11}=\frac{C_{1}^{2}}{C_{2}^{2}} \frac{\xi_{1}^{-}}{\xi_{1}^{+}} ; W_{1 j}=$ $\left(\frac{C_{1}^{2}}{C_{2}^{2}}-1\right) \frac{\xi_{j}}{\xi_{1}^{+}} ; W_{j j}=1, j \geq 2$; all the other entries are zero.

REMARK 3.3.

1. In (3.13), $\overrightarrow{e_{1}}$ is a column vector, and $\left[|\boldsymbol{\xi}| \nabla_{\boldsymbol{y}} c(\boldsymbol{y}) \cdot \nabla_{\boldsymbol{\xi}} \boldsymbol{\phi}^{T}\right]_{y_{1}=0}$ is a row vector, hence their multiplication produces an $n \times n$ matrix.

2. $W=\left(G^{\mathrm{T}}\right)^{-1}$ and $W^{\mathrm{T}}=G^{-1}$.

Combining (2.7), (3.11), and (3.13) yields the interface condition at $y_{1}=0$ for the transmitted beam,

$$
M_{3}=W M_{1} W^{\mathrm{T}}-\frac{\left|\boldsymbol{\xi}^{+}\right|^{2}}{C_{2} \xi_{1}^{+}} \overrightarrow{e_{1}}\left(\nabla_{\boldsymbol{y}} c_{2}(\boldsymbol{y})\right)^{\mathrm{T}}+\frac{\left|\boldsymbol{\xi}^{+}\right|\left|\boldsymbol{\xi}^{-}\right|}{C_{2} \xi_{1}^{+}} \overrightarrow{e_{1}}\left(\nabla_{\boldsymbol{y}} c_{1}(\boldsymbol{y})\right)^{\mathrm{T}} W^{\mathrm{T}}
$$

The interface condition (3.14) consists of two parts. The first part $W M_{1} W^{\mathrm{T}}$ is due to the jump of the wave speed. The other part originates from the jump of the first derivative of the wave speed.

REMARK 3.4.

1. In [27], Weiss and Hagedorn studied the problem when the wave speed was piecewise constant. Straightforward calculations show that the formulations (3.10) and (3.14) are consistent with their rigorous results.

2. Compared to the direct derivation of interface conditions in the Lagrangian framework (see [32] for example), the derivation from the Eulerian framework seems simpler.

\section{Numerical examples}

In this section, we give two examples to show the performance of the Eulerian Gaussian beam method. In order to show the asymptotic convergence, we eliminate the numerical errors by analytically solving the level set functions whenever possible.

EXAMPLE 4.1. We consider

$$
u_{t t}-c^{2}(x) u_{x x}=0, \quad u_{0}(x)=u(x, 0)=A_{0}(x) e^{i S_{0}(x) / \varepsilon}, \quad u_{t}(x, 0)=0,
$$

where

$$
A_{0}(x)=\mathrm{I}_{\{x<0\}} \exp \left(-25(x+0.8)^{2}\right), \quad S_{0}(x)=-x^{2} .
$$

The wave speed is taken as

$$
c(x)=\left\{\begin{array}{lr}
2 & x<0, \\
1.5+x / 6 & x>0 .
\end{array}\right.
$$




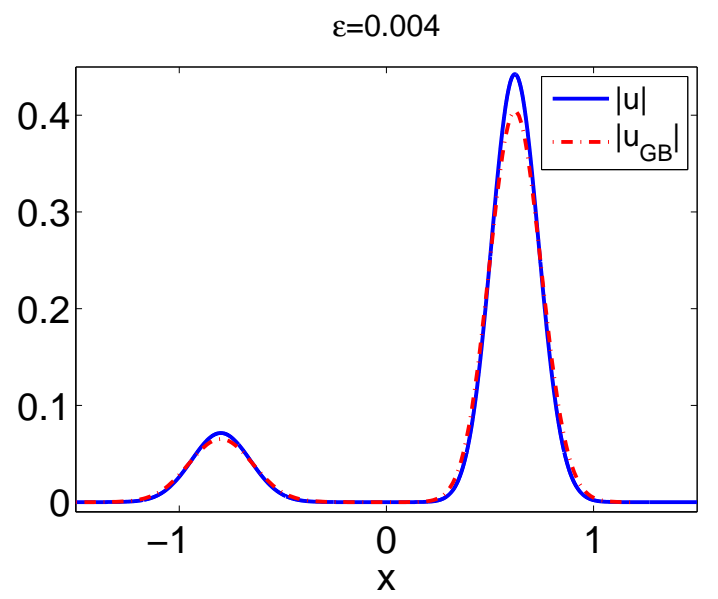

Fig. 4.1: Example 4.1, the comparison of the wave amplitudes of the reference solution and the Gaussian beam solution for $\epsilon=0.004$ at $t=0.8$.

In this example, both the wave speed and its first derivative have a jump at $x=0$. The reflection coefficient is $R=-1 / 7$ and transmission coefficient is $T=6 / 7$. We compare the reference solution and the Gaussian beam solution at $t=0.8$. The reference solution is obtained by the second order finite difference method using $N_{x}=2^{15}$ and $N_{t}=2^{19}$ for $x \in[-3.5,1.5]$. We take $\mathrm{d} y=\mathrm{d} \xi=0.007$ in the Gaussian beam method. We show the comparison of the wave amplitudes for $\epsilon=0.004$ in figure 4.1. We list the $l^{\infty}$ errors of the wave functions for different values of $\epsilon$ in table 4.1. The numerical convergence rate is 0.85 .

\begin{tabular}{ccccc}
\hline$\epsilon$ & 0.016 & 0.008 & 0.004 & 0.0002 \\
$\left\|u-u_{G B}\right\|_{\infty}$ & 0.11314 & 0.06892 & 0.03859 & 0.01907 \\
\hline
\end{tabular}

TABLE 4.1. Example 4.1, the $l^{\infty}$ errors of the wave functions for different values of $\epsilon$.

EXAMPLE 4.2. We consider

$$
u_{t t}-c^{2}(\boldsymbol{x}) \Delta_{\boldsymbol{x}} u=0, \quad u_{0}(\boldsymbol{x})=A_{0}(\boldsymbol{x}) e^{i S_{0}(\boldsymbol{x}) / \varepsilon}, \quad \partial_{t} u(\boldsymbol{x})=0, \quad \boldsymbol{x} \in \mathbb{R}^{3},
$$

where

$$
A_{0}(\boldsymbol{x})=\mathrm{I}_{\{0<|\boldsymbol{x}|<2\}} \exp \left(-25(|\boldsymbol{x}|-1.2)^{2}\right) /|\boldsymbol{x}|, \quad S_{0}(\boldsymbol{x})=-(|\boldsymbol{x}|-2)^{2} .
$$

The wave speed is given by

$$
c(\boldsymbol{x})= \begin{cases}2 & |\boldsymbol{x}| \leq 2 \\ 1.5 & |\boldsymbol{x}|>2 .\end{cases}
$$

In this example, the interface is placed at the radius direction. By Snell's Law of Refraction, the reflection coefficient is $R=-1 / 7$ and transmission coefficient is $T=6 / 7$. We compare the exact solution and the Gaussian beam solution at $t=0.8$. 
(a)

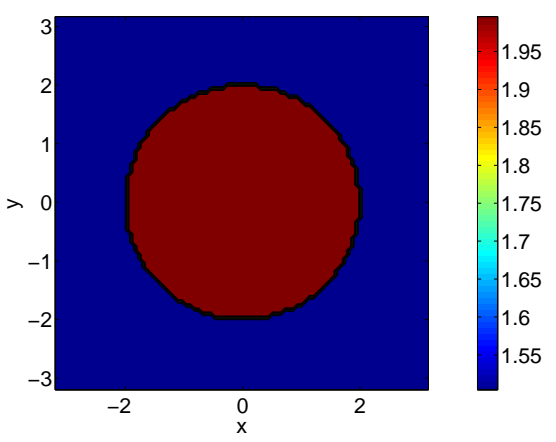

$(c)$

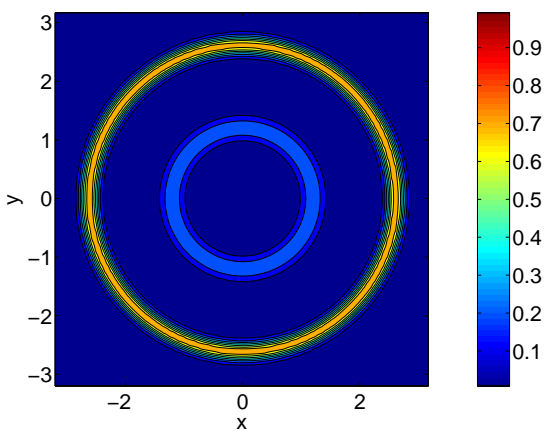

(b)

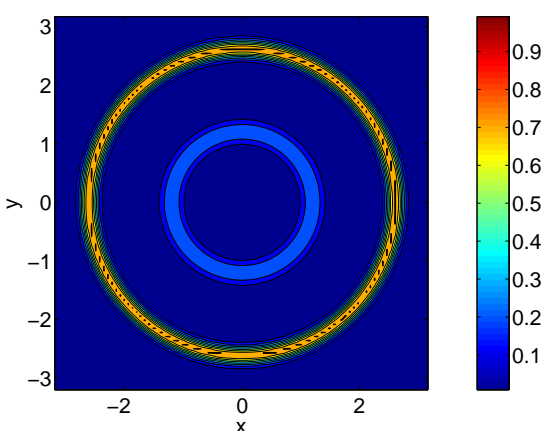

$(d)$

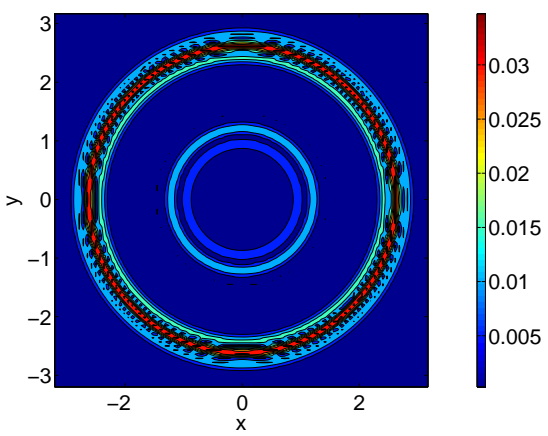

Fig. 4.2: Example 4.2, the comparison of the amplitudes of the reference solution and the Gaussian beam solution on the $x-y$ plane for $\epsilon=0.002$ at $t=0.8$. Figure (a) describes the local wave speed $c(\boldsymbol{x})$; figure (b) shows the reference solution $|u|$; figure (c) shows the Gaussian beam solution $\left|u_{G B}\right|$; figure (d) gives the error between them $\left|u-u_{G B}\right|$.

We show the comparison of the wave amplitudes on the $x-y$ plane for $\epsilon=0.002$ in figure 4.2. One can see the error is approximately at the order of $\sqrt{\varepsilon}$. We take 0.14 as the mesh size for $\boldsymbol{y}$ and $\boldsymbol{\xi}$ in the Gaussian beam method, and 0.02 as the mesh size for the reference solution.

\section{Concluding remarks}

In this paper, we generalize the Eulerian Gaussian beam method developed in [8] to compute the high frequency wave propagation in heterogeneous media with discontinuity in one direction. Motivated by the Hamiltonian preserving scheme developed in [6], we propose interface conditions to capture the reflected and transmitted Gaussian beams. We also give the equivalent interface conditions in the Lagrangian formulation. Especially we show that they are consistent with the rigorous results given in [27]. Several numerical examples are presented to verify the accuracy of this method.

Acknowledgment. Both authors thank Shi Jin for valuable discussions. D.W. was partially supported by the NSF grant DMS-0608720, and the NSF FRG grant DMS-0757285. X.Y. was partially supported by the DOE grant DE-SC0002618. 
[1] V. Cerveny, M.M. Popov, and I. Psencik, Computation of wave fields in inhomogeneous media - Gaussian beam approach, Geophys. J. Roy. Astr. Soc., 70, 109-128, 1982.

[2] B. Engquist and O. Runborg, Computational high frequency wave propagation, Acta Numer., $12,181-266,2003$.

[3] N.R. Hill, Gaussian beam migration, Geophys., 55, 1416-1428, 1990.

[4] N.R. Hill, Prestack Gaussian-beam depth migration, Geophys., 66, 1240-1250, 2001.

[5] S. Jin, P.A. Markowich, and C. Sparber, Mathematical and computational methods for semiclassical Schrödinger equations, Acta Numer., 20, 211-289, 2011.

[6] S. Jin and X. Wen, Hamiltonian-preserving schemes for the Liouville equation with discontinuous potentials, Commun. Math. Sci., 3, 285-315, 2005.

[7] S. Jin, H. Wu, and Z.Y. Huang, A hybrid phase-flow method for Hamiltonian systems with discontinuous Hamiltonians, SIAM J. Sci. Comput., 31, 1303-1321, 2008.

[8] S. Jin, H. Wu, and X. Yang, Gaussian beam methods for the Schrödinger equation in the semi-classical regime: Lagrangian and Eulerian Formulations, Commun. Math. Sci., 6, 995-1020, 2008.

[9] S. Jin, H. Wu, X. Yang, and Z. Huang, Bloch decomposition-based Gaussian beam method for the Schrödinger equation with periodic potentials, J. Comput. Phys., 229, 4869-4883, 2010.

[10] S. Jin, H. Wu, and X. Yang, A numerical study of the Gaussian beam methods for onedimensional Schrödinger-Poisson equations, J. Comput. Math., 28, 261-272, 2010.

[11] S. Jin, H. Wu, and X. Yang, Semi-Eulerian and high order Gaussian beam methods for the Schrödinger equation in the semiclassical regime, Commun. Comput. Phys., 9, 668-687, 2011.

[12] S. Leung, J. Qian, and R. Burridge, Eulerian Gaussian beams for high-frequency wave propagation, Geophys., 72, 61-76, 2007.

[13] S. Leung and J. Qian, Eulerian Gaussian beams for Schrödinger equations in the semiclassical regime, J. Comput. Phys., 228, 2951-2977, 2009.

[14] H. Liu and J. Ralston, Recovery of high frequency wave fields for the acoustic wave equation, Multiscale Model. Simul., 8, 428-444, 2009.

[15] H. Liu and J. Ralston, Recovery of high frequency wave fields from phase space based measurements, Multiscale Model. Simul., 8, 622-644, 2010.

[16] J. Lu and X. Yang, Frozen Gaussian approximation for high frequency wave propagation, Commun. Math. Sci., 9, 663-683, 2011.

[17] J. Lu and X. Yang, Frozen Gaussian approximation for general linear strictly hyperbolic systems: Formulation and Eulerian methods, Multiscale Model. Simul., Multiscale Model. Simul., 10, 451-472, 2012.

[18] J. Lu and X. Yang, Convergence of frozen Gaussian approximation for high frequency wave propagation, Commun. Pure Appl. Math., 65, 759-789, 2012.

[19] M. Motamed and O. Runborg, Taylor expansion and discretization errors in Gaussian beam superposition, Wave Motion, 47, 421-439, 2010.

[20] M.M. Popov, A new method of computation of wave fields using Gaussian beams, Wave Motion, 4, 85-97, 1982.

[21] J. Qian and L. Ying, Fast Gaussian wavepacket transforms and Gaussian beams for the Schrödinger equation, J. Comput. Phys., 229, 7848-7873, 2010.

[22] J. Qian and L. Ying, Fast multiscale Gaussian wavepacket transforms and multiscale Gaussian beams for the wave equation, Multiscale Model. Simul., 8, 1803-1837, 2010.

[23] J. Ralston, Gaussian beams and the propagation of singularities, Studies in PDEs, MAA Stud. Math., 23, 206-248, 1982.

[24] N.M. Tanushev, J. Qian, and J. Ralston, Mountain waves and Gaussian beams, Multiscale Model. Simul., 6, 688-709, 2007.

[25] N.M. Tanushev, Superpositions and higher order Gaussian beams, Commun. Math. Sci., 6, 449-475, 2008.

[26] D. Wei, S. Jin, R. Tsai, and X. Yang, A level set method for the semiclassical limit of the Schrödinger equation with discontinuous potentials, J. Comput. Phys., 229, 7440-7455, 2010.

[27] W.R.E. Weiss and G.A. Hagedorn, Reflection and transmission of high freuency pulses at an interface, Transport Theory Statist. Phys., 14, 539-565, 1985.

[28] X. Wen, High order numerical quadratures to one dimensional delta function integrals, SIAM J. Sci. Comput. 30, 1825-1846, 2008.

[29] X. Wen, High order numerical methods to two dimensional delta function integrals in level set methods, J. Comput. Phys. 228, 4273-4290, 2009.

[30] X. Wen, High order numerical methods to three dimensional delta function integrals in level set methods, SIAM J. Sci. Comput. 32, 1288-1309, 2010. 
[31] H. Wu and X. Yang, A hybrid phase-flow method for solving the Liouville equation in bounded domain, SIAM J. Numer. Anal., 49, 733-754, 2011.

[32] D. Yin and C. Zheng, Gaussian beam formulations and interface conditions for the onedimensional linear Schrödinger equation, Wave Motion, 48, 310-324, 2011. 